\title{
Dynamical Simulations with Highly Improved Staggered Quarks
}

\author{
K. Y. Wong \\ Department of Physics \& Astronomy, University of Glasgow, Glasgow, UK G12 8QQ \\ E-mail: k.wongephysics.gla.ac.uk
}

\section{R. M. Woloshyn*}

TRIUMF, 4004 Wesbrook Mall, Vancouver, British Columbia, Canada V6T 2A3

E-mail: rwwwetriumf.ca

It is well established that lattice artifacts can be suppressed substantially by the use of SU(3)projected smeared links in the fermion action. An example is the Highly Improved Staggered Quark action where the ASQ-like effective links are constructed from reunitarized Fat7 links. A general procedure is presented for computing the derivative of the fermion action with respect to the base links (fermion force) - a key component in dynamical simulations using molecular dynamics evolution. The method is iterative and can be applied to actions with arbitrary levels of smearing and reunitarization. The cost of calculating the fermion force is determined for the ASQ action and the HISQ action. Test results show that calculating the HISQ force is about two times more expensive than the ASQ force.

The XXV International Symposium on Lattice Field Theory

July 30 - August 42007

Regensburg, Germany

\footnotetext{
* Speaker.
} 


\section{Introduction}

The staggered quark formalism provides a fast method to simulate light quarks at small quark masses. Staggered fermions, however, have the property that each lattice quark field describes four identical quark flavors ("tastes") rather than one, and coupling to gauge fields leads to tastechanging errors, which are $O\left(a^{2}\right)$ effects [1]. Taste-changing interactions can be suppressed substantially by the use of fat links [1, 2, 3, 包; accurate simulation results [5, 6, 7] were obtained using the ASQTAD action, where the base links are replaced by Fat7 effective links (see Fig. 1). The success of the ASQTAD action suggests that further improvement may be possible with more smearing. Perturbative calculations and non-perturbative results of Refs. [8, 9] show that significant improvement can be obtained by first projecting the fat links back to SU(3) matrices before being used in the next fattening process. The result is the Highly Improved Staggered Quark action (HISQ) where the ASQ-like effective links are constructed from reunitarized Fat7 links (another widely used smearing technique is HYP-smearing [10], which is based on SU(3)-projected fat links also)

$$
U^{e f f}\left(U^{R}, U^{R \dagger}\right), \quad U^{R}\left(U^{F}, U^{F \dagger}\right), \quad U^{F}\left(U, U^{\dagger}\right) .
$$

Here $U$ is the base link, $U^{F}$ is the Fat7 link, $U^{R}$ is the reunitarized fat-link and $U^{e f f}$ is the ASQlike effective link (see Fig. 1). The HISQ action has no tree-level order $a^{2}$ errors like the ASQTAD action, and has taste-changing interactions that are 3-4 times smaller than the ASQTAD action. In addition, the action has no tree-level order $(\mathrm{am})^{4}$ errors to leading order in the quark's velocity $v / c$, it therefore provides an accurate discretization of the charm quark on the lattice. For example this action has been used recently to obtain high precision results for $D$ meson and decay constants for $\pi, K, D$ and $D_{s}$ [9, 11].

So far all unquenched simulations using staggered quarks as light quarks were done with the ASQTAD action. Given the nice features of the HISQ action, it is desirable to use it for the sea quarks also. Dynamical simulations with HISQ, however, are complicated by the extra level of fattening and $\mathrm{SU}(3)$-projection. In this paper we present a general procedure for computing the derivative of the fermion action with respect to the base links (fermion force) - a key component in dynamical simulations using molecular dynamics evolution. This method is iterative and can be applied to actions with arbitrary levels of smearing or SU(3)-projection. We compare the efficiency of the algorithm for the ASQTAD and HISQ actions on small lattices.

Our treatment of unitarized links have been influenced by Kamleh et al. [12]. Other approaches have been discussed in Refs. [13, 14, 15].

\section{The Fermion Force}

The staggered quark action is (we follow the notations of [16])

$$
S_{f}=\left\langle\Phi\left|\left[M^{\dagger}[U] M[U]\right]^{-n_{f} / 4}\right| \Phi\right\rangle,
$$

where

$$
\begin{aligned}
M_{x, y}[U] & =2 m \delta_{x, y}+D_{x, y}[U] \\
& =2 m \delta_{x, y}+\sum_{\mu} \eta_{x, \mu}\left(U_{x, \mu}^{e f f} \delta_{x, y-\mu}-U_{x-\mu, \mu}^{e f f \dagger} \delta_{x, y+\mu}\right) .
\end{aligned}
$$




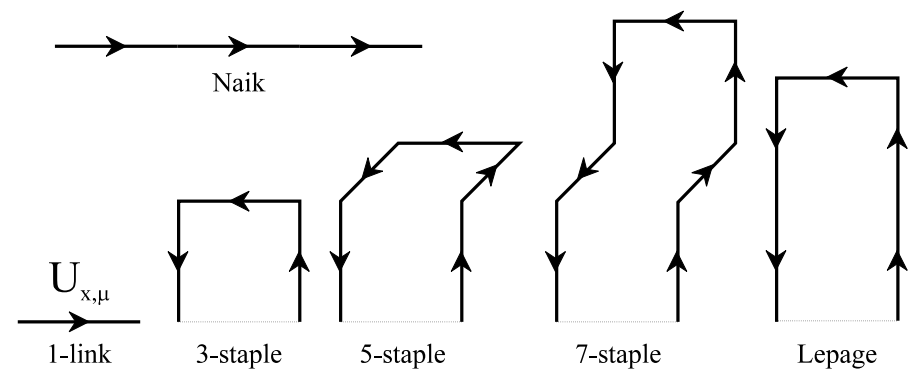

Figure 1: Paths used in the ASQTAD and HISQ actions. Path coefficients for ASQTAD can be found in Ref. [4]. The HISQ effective links are constructed by first applying a Fat7 fattening to the base links $\left(U \rightarrow U^{F}\right.$ with coefficients 1-link:1/8, 3-staple:1/16, 5-staple:1/64, 7-staple:1/384), then a SU(3)projection $\left(U^{F} \rightarrow U^{R}\right)$, and finally an "ASQ" smearing $\left(U^{R} \rightarrow U^{\text {eff }}\right.$ with coefficients 1-link:1 $+\varepsilon / 8$, 3staple:1/16, 5-staple:1/64, 7-staple:1/384, Lepage:-1/8, Naik:- $(1+\varepsilon) / 24$, where the parameter $\varepsilon$ is introduced to remove $(\mathrm{am})^{4}$ errors [9]).

The pseudo-fermion field $\Phi$ is defined on even lattice sites only to avoid a doubling of flavors from using $M^{\dagger} M$ instead of $M$ in the action. This procedure is valid since $M^{\dagger} M$ has no matrix element connecting even and odd lattice sites.

A key component in dynamical simulations using molecular dynamics evolution is the computation of fermion force - derivative of the fermion action with respect to the base links

$$
f_{x, \mu}=\frac{\partial S_{f}}{\partial U_{x, \mu}}=\frac{\partial}{\partial U_{x, \mu}}\left\langle\Phi\left|\left[M^{\dagger}[U] M[U]\right]^{-n_{f} / 4}\right| \Phi\right\rangle .
$$

The derivative can be computed straightforwardly if $n_{f}$ is a multiple of 4 ; for other numbers of fermion flavors the 4th-root of $M^{\dagger} M$ can be approximated by a rational expansion (the RHMC algorithm [17, 18, 19])

$$
\left[M^{\dagger} M\right]^{-n_{f} / 4} \approx \alpha_{0}+\sum_{l} \frac{\alpha_{l}}{M^{\dagger} M+\beta_{l}},
$$

where $\alpha_{l}$ and $\beta_{l}$ are constants. The derivative becomes

$$
\begin{aligned}
\frac{\partial S_{f}}{\partial U_{x, \mu}} & =-\sum_{l} \alpha_{l}\left\langle\Phi\left[M^{\dagger} M+\beta_{l}\right]^{-1}\left|\frac{\partial}{\partial U_{x, \mu}}\left(M^{\dagger}[U] M[U]\right)\right|\left[M^{\dagger} M+\beta_{l}\right]^{-1} \Phi\right\rangle \\
& =-\sum_{l} \alpha_{l}\left(\left\langle X^{l}\left|\frac{\partial D^{\dagger}[U]}{\partial U_{x, \mu}}\right| Y^{l}\right\rangle+\left\langle Y^{l}\left|\frac{\partial D[U]}{\partial U_{x, \mu}}\right| X^{l}\right\rangle\right),
\end{aligned}
$$

with $\left|X^{l}\right\rangle=\left[M^{\dagger} M+\beta_{l}\right]^{-1}|\Phi\rangle$ and $\left|Y^{l}\right\rangle=D\left|X^{l}\right\rangle$. Note that $X^{l}$ and $Y^{l}$ are defined on even and odd sites respectively. Taking the derivatives of $D, D^{\dagger}$ with respect to $U^{e f f}, U^{e f f \dagger}$ and writing out the matrix indices we have

$$
\left[f_{x, \mu}\right]_{a b}=\frac{\partial S_{f}}{\partial\left[U_{x, \mu}\right]_{a b}}=\sum_{y, v}(-1)^{y} \eta_{y, v}\left(\frac{\partial\left[U_{y, v}^{e f f}\right]_{m n}}{\partial\left[U_{x, \mu}\right]_{a b}}\left[f_{y, v}^{(0)}\right]_{m n}+\frac{\partial\left[U_{y, v}^{e f f \dagger}\right]_{m n}}{\partial\left[U_{x, \mu}\right]_{a b}}\left[f_{y, v}^{(0) \dagger}\right]_{m n}\right),
$$

where $f_{y, v}^{(0)}$ is the vector outer product of the field variables at $y$ and $y+v$

$$
\left[f_{y, v}^{(0)}\right]_{m n}=\left\{\begin{array}{ll}
\sum_{l} \alpha_{l}\left[Y_{y+v}^{l}\right]_{n}\left[X_{y}^{l *}\right]_{m} & \text { for even } y \\
\sum_{l} \alpha_{l}\left[X_{y+v}^{l}\right]_{n}\left[Y_{y}^{l *}\right]_{m} & \text { for odd } y
\end{array},\right.
$$


and the sum on $y, v$ extends over the effective links that contain the base link $U_{x, \mu}$, and $(-1)^{y}=1$ for even $y$ 's and $(-1)^{y}=-1$ for odd sites. Note that the force is equal to $f^{(0)}$ when there is no smearing, i.e., $U^{e f f}=U$. Furthermore all complications associated with the rational expansion have been absorbed into $f^{(0)}$ and the derivatives $\partial U^{e f f} / \partial U, \partial U^{e f f \dagger} / \partial U$ are calculated only once.

\section{Smearing and SU(3)-Projection}

For actions with multiple levels of smearing or SU(3)-projection, such as the HISQ action, we use the chain rule to compute $\partial U^{e f f} / \partial U, \partial U^{e f f \dagger} / \partial U$

$$
\begin{aligned}
{\left[f_{x, \mu}\right]_{a b}=\sum_{y, v ; z, \rho}(-1)^{y} \eta_{y, v}\{} & \left(\frac{\partial\left[U_{y, v}^{e f f}\right]_{m n}}{\partial\left[U_{z, \rho}^{R}\right]_{p q}} \frac{\partial\left[U_{z, \rho}^{R}\right]_{p q}}{\partial\left[U_{x, \mu}\right]_{a b}}+\frac{\partial\left[U_{y, v}^{e f f}\right]_{m n}}{\partial\left[U_{z, \rho}^{R \dagger}\right]_{p q}} \frac{\partial\left[U_{z, \rho}^{R^{\dagger}}\right]_{p q}}{\partial\left[U_{x, \mu}\right]_{a b}}\right)\left[f_{y, v}^{(0)}\right]_{m n} \\
+ & \left.\left(U^{e f f} \rightarrow U^{e f f \dagger}\right)\left[f_{y, v}^{(0) \dagger}\right]_{m n}\right\} \\
= & \sum_{z, \rho}\left(\frac{\partial\left[U_{z, \rho}^{R}\right]_{p q}}{\partial\left[U_{x, \mu}\right]_{a b}}\left[f_{z, \rho}^{(1)}\right]_{p q}+\frac{\partial\left[U_{z, \rho}^{R \dagger}\right]_{p q}}{\partial\left[U_{x, \mu}\right]_{a b}}\left[f_{z, \rho}^{(1) \dagger}\right]_{p q}\right)
\end{aligned}
$$

where the sum on $z, \rho$ extends over the reunitarized links $U^{R}$ that contain the base link $U_{x, \mu}$, and

$$
\left[f_{z, \rho}^{(1)}\right]_{p q}=\sum_{y, v}(-1)^{y} \eta_{y, v}\left(\frac{\partial\left[U_{y, v}^{e f f}\right]_{m n}}{\partial\left[U_{z, \rho}^{R}\right]_{p q}}\left[f_{y, v}^{(0)}\right]_{m n}+\frac{\partial\left[U_{y, v}^{e f f \dagger}\right]_{m n}}{\partial\left[U_{z, \rho}^{R}\right]_{p q}}\left[f_{y, v}^{(0) \dagger}\right]_{m n}\right) .
$$

The expression for $f_{x, \mu}$ still contains the composite derivative $\partial U^{R} / \partial U$. Repeat this step until all the derivatives are explicit

$$
\begin{gathered}
{\left[f_{z, \rho}^{(2)}\right]_{r s}=\frac{\partial\left[U_{z, \rho}^{R}\right]_{p q}}{\partial\left[U_{z, \rho}^{F}\right]_{r s}}\left[f_{z, \rho}^{(1)}\right]_{p q}+\frac{\partial\left[U_{z, \rho}^{R \dagger}\right]_{p q}}{\partial\left[U_{z, \rho}^{F}\right]_{r s}}\left[f_{z, \rho}^{(1) \dagger}\right]_{p q}} \\
{\left[f_{x, \mu}\right]_{a b}=\left[f_{x, \mu}^{(3)}\right]_{a b}=\sum_{z, \rho}\left(\frac{\partial\left[U_{z, \rho}^{F}\right]_{r s}}{\partial\left[U_{x, \mu}\right]_{a b}}\left[f_{z, \rho}^{(2)}\right]_{r s}+\frac{\partial\left[U_{z, \rho}^{F \dagger}\right]_{r s}}{\partial\left[U_{x, \mu}\right]_{a b}}\left[f_{z, \rho}^{(2) \dagger}\right]_{r s}\right) .}
\end{gathered}
$$

Note that $f^{(2)}$ is local since $U_{z, \rho}^{R}$ is a function of $U_{z, \rho}^{F}, U_{z, \rho}^{F \dagger}$ only. Therefore to construct the fermion force one starts with $f^{(0)}$, computes $f^{(i)}$ for each smearing and $\mathrm{SU}(3)$-projection working in towards the first level of smearing. This procedure is very general and can be applied to actions with arbitrary levels of smearing and reunitarization.

Two types of derivatives are involved: derivatives of smeared links ( $\partial U^{e f f} / \partial U^{R}$ and $\partial U^{F} / \partial U$ ) and derivatives of reunitarized links $\left(\partial U^{R} / \partial U^{F}\right)$. Implementing the smeared links and their derivatives is relatively straightforward. For SU(3)-projected smeared links we use polar decomposition since it is differentiable and therefore suitable for dynamical simulations (another popular choice is the stout link introduced by Morningstar and Peardon [13])

$$
U^{R}=\frac{\tilde{U}^{R}}{\left(\operatorname{det} \tilde{U}^{R}\right)^{1 / 3}}, \quad \tilde{U}^{R}=U^{F}\left[U^{F \dagger} U^{F}\right]^{-1 / 2}
$$


There are several ways to compute the derivative $\partial U^{R} / \partial U^{F}$. Refs. [13, 15] utilize the CayleyHamilton Theorem; in Ref. [9] the derivative is obtained by solving a matrix equation. Here we follow Ref. [12] by adopting a rational expansion for $\left[U^{F^{\dagger}} U^{F}\right]^{-1 / 2}$

$$
\left[U^{F \dagger} U^{F}\right]^{-1 / 2}=c_{0}+\sum_{l} \frac{c_{l}}{U^{F \dagger} U^{F}+d_{l}},
$$

where $c_{l}$ and $d_{l}$ are constants. A nice feature of the rational approximation is that the derivatives of $U^{R}$ are calculated easily

$$
\begin{aligned}
\frac{\partial\left[\tilde{U}^{R}\right]_{p q}}{\partial\left[U^{F}\right]_{r s}} & =\delta_{p r}\left[c_{0}+\sum_{l} \frac{c_{l}}{U^{F \dagger} U^{F}+d_{l}}\right]_{s q} \\
& -\sum_{l} c_{l}\left[U^{F} \frac{1}{U^{F \dagger} U^{F}+d_{l}} U^{F \dagger}\right]_{p r}\left[\frac{1}{U^{F \dagger} U^{F}+d_{l}}\right]_{s q},
\end{aligned}
$$

and including the determinant

$$
\frac{\partial\left[U^{R}\right]_{p q}}{\partial\left[U^{F}\right]_{r s}}=\left(\operatorname{det} \tilde{U}^{R}\right)^{-1 / 3}\left\{-\frac{1}{3} \operatorname{tr}\left(\tilde{U}^{R^{-1}} \frac{\partial \tilde{U}^{R}}{\partial\left[U^{F}\right]_{r s}}\right)\left[\tilde{U}^{R}\right]_{p q}+\frac{\partial\left[\tilde{U}^{R}\right]_{p q}}{\partial\left[U^{F}\right]_{r s}}\right\}
$$

where the trace is taken with respect to the indices on $\tilde{U}^{R}$. Finally the derivative can also be computed numerically

$$
\frac{\partial\left[U^{R}\right]_{p q}}{\partial\left[U^{F}\right]_{r s}}=\frac{\left[U^{R}\right]_{p q}\left(\left[U^{F}\right]_{r s}+h\right)-\left[U^{R}\right]_{p q}\left(\left[U^{F}\right]_{r s}-h\right)}{2 h},
$$

with $U^{F \dagger}$ being held fixed. The matrices $U^{R}\left(U^{F} \pm h\right)$ can be obtained by diagonalizing $U^{F} U^{F \dagger}$. This method, however, is relatively inefficient compared to the rational approximation approach (typically by a factor of five). On the other hand, it provides a useful check on our approximate calculation.

\section{Benchmark}

We test the performance of the HISQ force on small lattices with a scalar code. We first compare the average plaquettes obtained using the RHMC algorithm and the R-algorithm. A high acceptance rate provides an excellent check of the code. Results are shown in Fig. 2.

Fig. 3a compares the cost of different components for the ASQTAD action and the HISQ action at different lattice volumes. Results show that matrix inversions (with no optimization) dominate for ASQTAD while the computation of fermion force and inversions are comparable for HISQ. It is interesting to note that the cost of calculating $f^{(2)}$, derivatives of reunitarized links, is relatively small compared to $f^{(1)}$ and $f^{(3)}$, derivatives of smeared links. This implies that the HISQ force is only about twice as expensive as the ASQ force. This point is further emphasized in Fig. 3b where the ratio of the cost of fermion force to the cost of gauge force is plotted for the two actions. Finally, the insensitivity of our results to lattice volume suggests that Fig. 3 is valid for larger lattices also. 


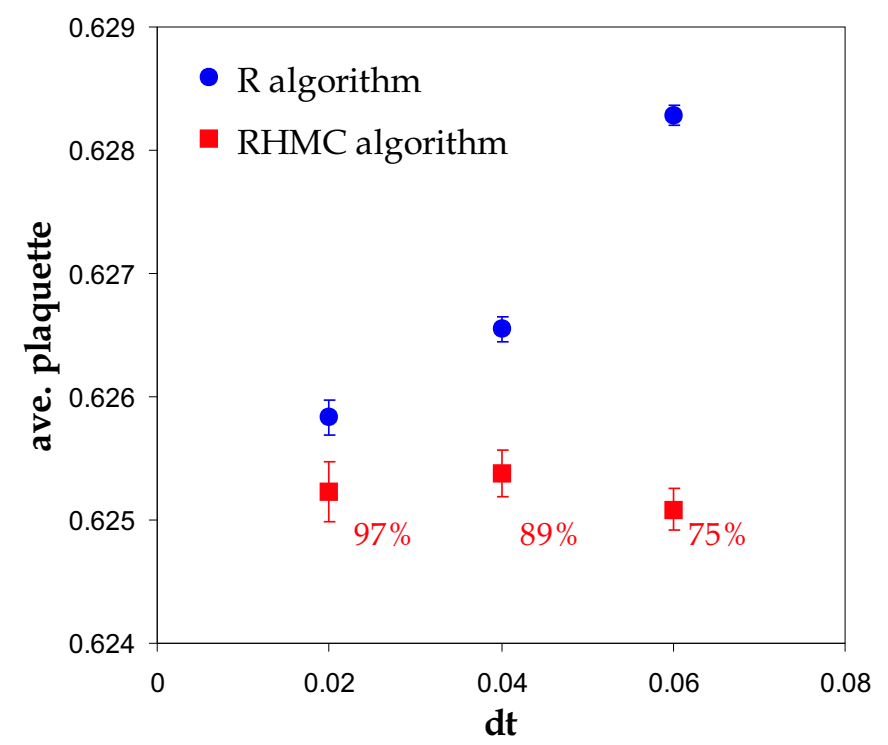

Figure 2: Average plaquettes obtained using the RHMC algorithm and the R-algorithm for the HISQ action (with $\varepsilon=1$ ). Simulation parameters are $\beta_{p l}=8.0, n_{f}=2$, am $=0.25, V=4^{4}$ and $n_{m d} \times d t \sim O(1)$ where $d t=0.02,0.04,0.06$ is the molecular dynamics step size and $n_{m d}=50,25,16$ is the number of steps. The 1-loop Symanzik-improved gluon action is used with tadpole factor $u_{0}=0.8897$. The RHMC acceptance rates are shown in red.

(a) cost of different components per trajectory

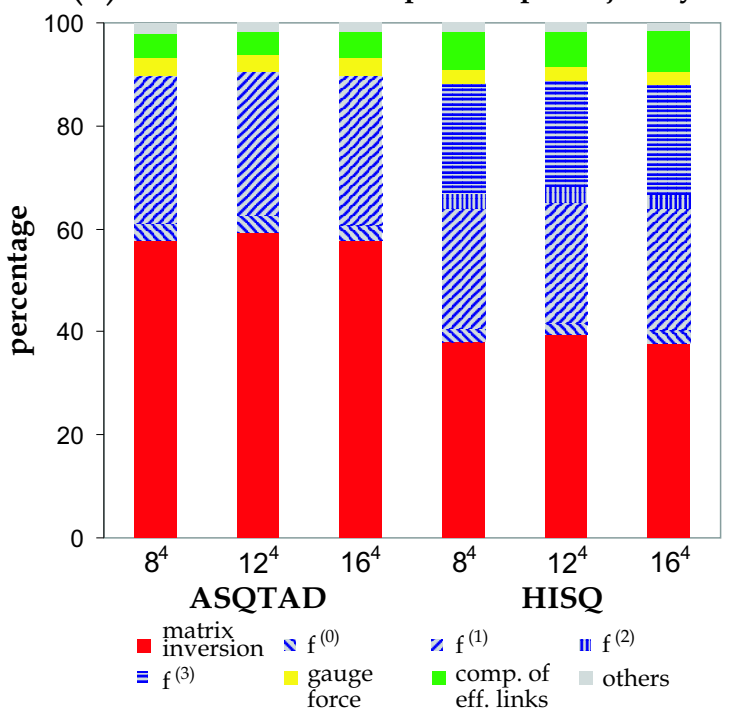

(b) cost of fermion force/ cost of gauge force

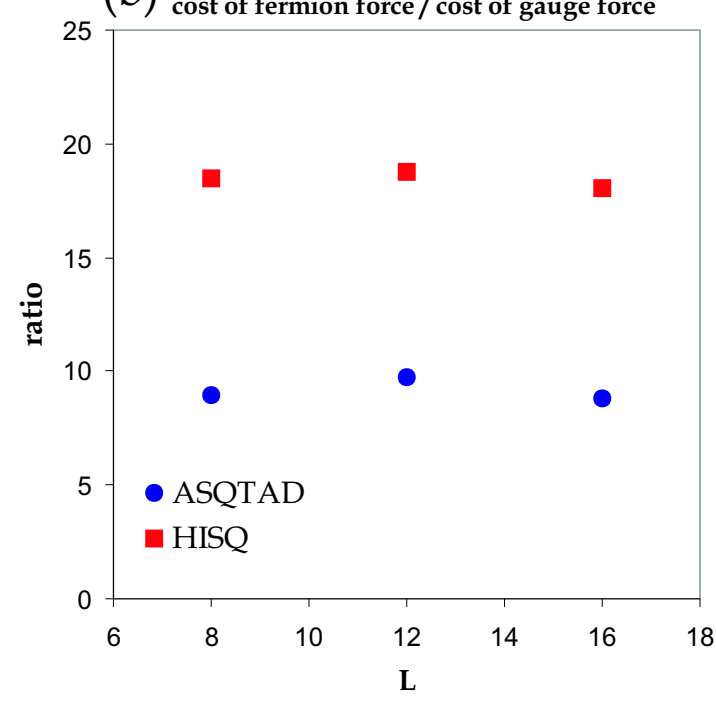

Figure 3: a) Cost of different components: $f^{(0)}$ is the force when there is no smearing, $f^{(1)}$ and $f^{(3)}$ are the derivatives of smeared links and $f^{(2)}$ is the derivative of reunitarized links. Simulation parameters are the same as those in Fig. 2 and the RHMC algorithm is used. b) Ratio of the cost of fermion force to the cost of gauge force for ASQTAD and HISQ. 


\section{Conclusion}

A general procedure is presented for computing the fermion force for actions constructed with SU(3)-projected smeared links. Application to the HISQ action has been discussed and tests have been done on small lattices. Our results show that the HISQ force is only two times more expensive than the ASQ force, with most of the additional cost attributed to the extra level of Fat7-smearing. Given that it is relatively inexpensive to compute the fermion force one should seriously consider using SU(3)-projected smeared links in future simulations.

\section{Acknowledgments}

We are very grateful to all our collaborators, and particularly Kent Hornbostel, for many useful discussions. Computations were done on facilities provided by WestGrid. This work was supported in part by the Natural Science and Engineering Research Council of Canada, and the Particle Physics and Astronomy Research Council of the UK.

\section{References}

[1] G. P. Lepage, Phys. Rev. D59:074502 (1999) [arXiv:hep-lat/9809157].

[2] K. Orginos et al., Phys. Rev. D59:014501 (1999) [arXiv:hep-lat/9805009].

[3] J. F. Lagae and D. K. Sinclair, Phys. Rev. D59:014511 (1999) [arXiv:hep-lat/9806014].

[4] K. Orginos, D. Toussaint and R. L. Sugar, Phys. Rev. D60:054503 (1999) [arXiv:hep-lat/9903032].

[5] C. T. H. Davies et al., Phys. Rev. Lett.92:022001 (2004) [arXiv:hep-lat/0304004].

[6] C. Aubin et al., Phys. Rev. D70:114501 (2004) [arXiv:hep-lat/0407028].

[7] A. S. Kronfeld et al., PoS LAT2005, 206 (2006) [arXiv:hep-lat/0610048]; J. Phys. Conf. Ser. 46:147 (2006) [arXiv:hep-lat/0607011].

[8] E. Follana et al., Nucl. Phys. Proc. Suppl. 129, 447 (2004) [arXiv:hep-lat/0311004].

[9] E. Follana et al., Phys. Rev. D75:054502 (2007) [arXiv:hep-lat/0610092].

[10] A. Hasenfratz and F. Knechtli, Phys. Rev. D64:034504 (2001) [arXiv:hep-lat/0103029].

[11] E. Follana et al., arXiv:hep-lat/0706.1726.

[12] W. Kamleh, D. B. Leinweber and A. G. Williams, Phys. Rev. D70:014502 (2004).

[13] C. Morningstar and M. J. Peardon, Phys. Rev. D69, 054501 (2004) [arXiv:hep-lat/0311018].

[14] T. A. DeGrand and S. Schaefer, Phys. Rev. D71, 034507 (2005) [arXiv:hep-lat/0412005].

[15] A. Hasenfratz, R. Hoffmann and S. Stefan, JHEP 0705:29 (2007) [arXiv:hep-lat/0702028].

[16] S. Gottlieb et al., Phys. Rev. D35, 2531 (1987).

[17] A. D. Kennedy, I. Horvath and S. Sint, Nucl. Phys. Proc. Suppl. 73, 834 (1999) [arXiv:hep-lat/9809092].

[18] M. A. Clark and A. D. Kennedy, Nucl. Phys. Proc. Suppl. 129, 850 (2004) [arXiv:hep-lat/0309084].

[19] M. A. Clark, PoS LAT2006, 4 (2006) [arXiv:hep-lat/0610048]. 